\title{
On smooth points of boundaries of open sets
}

\author{
by
}

\section{S. Rolewicz (Warszawa)}

\begin{abstract}
The notions of smooth points of the boundary of an open set and $\alpha(\cdot)$ intrinsically paraconvex sets are introduced. It is shown that for an $\alpha(\cdot)$ intrinsically paraconvex open set the set of smooth points is a dense $G_{\delta}$-set of the boundary.
\end{abstract}

Let $(X,\|\cdot\|)$ be a Banach space. Let $C$ be an open set in $X$ and let $x_{0} \in \bar{C}$.

The tangent cone $\mathcal{T}_{C}\left(x_{0}\right)$ of the set $C$ at the point $x_{0}$ consists of $h \in X$ such that for every neighbourhood $Q$ of $h$ there is $t_{Q}>0$ such that for $0<t<t_{Q}$,

$$
\left(x_{0}+t Q\right) \cap C \neq \emptyset
$$

(Dubovitskiı̌ and Milyutin (1965)). It is easy to see that each tangent cone is closed.

Using the distance function we can rewrite this definition in the following form: $\mathcal{T}_{C}\left(x_{0}\right)$ consists of $h \in X$ such that for every $\varepsilon>0$ there is a $t_{0}>0$ such that for $0<t<t_{0}$,

$$
\operatorname{dist}\left(x_{0}+t h, C\right)<\varepsilon t .
$$

Here the arbitrariness of $\varepsilon$ means that the directional derivative of the function $\operatorname{dist}(\cdot, C)$ at the point $x_{0}$ in the direction $h$ is equal to 0 , $\left.\partial \operatorname{dist}(x, C)\right|_{x_{0}}(h)=0$.

A point $x_{0} \in \partial C$ is called a smooth point of $\partial C$ if the cone $\mathcal{T}_{C}\left(x_{0}\right)$ is a halfspace, i.e.

$$
\mathcal{T}_{C}\left(x_{0}\right)=\left\{x \in X: x^{*}(x) \geq 0\right\}
$$

for some continuous linear functional $x^{*}$. The set of all smooth points of $\partial C$ is called the smooth set of the $\partial C$ and denoted by $\mathcal{S}(C)$.

It is a natural question how big part of $\partial C$ is the smooth set $\mathcal{S}(C)$.

If $\mathcal{S}(C)=\partial C$ we say that $\partial C$ is smooth.

Now we give an example of a smooth set $C$. 
Proposition 1. Let $(Y,\|\cdot\|)$ be a Banach space. Let $\Omega$ be an open set in $Y$. Let $C \subset \mathbb{R} \times \Omega$ be the epigraph of a function $f: \Omega \rightarrow \mathbb{R}, C=\{(r, y) \in$ $\mathbb{R} \times \Omega: r \geq f(y), y \in \Omega\}$. If $f$ is Gateaux differentiable at $y_{0}$ then $\left(f\left(y_{0}\right), y_{0}\right)$ is a smooth point of $\partial C$.

Proof. We take $X=\mathbb{R} \times Y$ and we put $x^{*}((t, y))=t+\left.\partial f\right|_{y_{0}}(y)$ and $H_{x^{*}}=\left\{(r, y): r \geq f\left(y_{0}\right)+\left.\partial f\right|_{y_{0}}(y)\right\}$. Since $f$ is Gateaux differentiable at $y_{0}$, for every $(r, y) \in H_{x^{*}}$ and every neighbourhood $Q$ of zero in $X$ there is $s>0$ such that for all $0<t<s$,

$$
\left(f\left(y_{0}\right), y_{0}\right)+t\left(r-f\left(y_{0}\right), y-y_{0}\right) \in C+t Q .
$$

Thus $H_{x^{*}} \subset \mathcal{T}_{C}\left(\left(f\left(y_{0}\right), y_{0}\right)\right)$.

On the other hand, if $(r, y) \notin H_{x^{*}}$ then for all $s>0$ there are $t_{s}>0$ and a neighbourhood $Q$ of zero such that $0<t_{s}<s$ and $\left(f\left(y_{0}\right), y_{0}\right)+$ $t_{s}\left(r-f\left(y_{0}\right), y-y_{0}\right) \notin C+t_{s} Q$. This implies that $(r, y) \notin \mathcal{T}_{C}\left(\left(f\left(y_{0}\right), y_{0}\right)\right)$. Therefore we have the equality

$$
H_{x^{*}}=\mathcal{T}_{C}\left(\left(f\left(y_{0}\right), y_{0}\right)\right)
$$

The converse assertion does not hold:

ExAmple 2. Let $Y=\mathbb{R}$. Let

$$
f(x)= \begin{cases}|x|\left(-1+\sin \frac{1}{x}\right) & \text { if } x \neq 0, \\ 0 & \text { if } x=0 .\end{cases}
$$

Of course, the function $f(\cdot)$ is not differentiable at 0 . On the other hand, the tangent cone of the epigraph $C=\{(t, x): t \geq f(x)\}$ at the point $(0,0)$ is a halfplane: $\mathcal{T}_{C}((0,0))=\{(t, x): t \geq 0\}$.

Mazur (1933) proved that if $X$ is separable, then every convex real-valued function defined on an open convex set $\Omega \subset X$ is Gateaux differentiable on a dense $G_{\delta}$-set. Of course, such sets are residual (i.e. their complements in $\Omega$ are of the first Baire category).

Asplund (1968) found a class of Banach spaces $X$ such that every convex real-valued function defined on an open convex set $\Omega \subset X$ is Fréchet differentiable on a dense $G_{\delta}$-set. Such spaces are now called Asplund spaces and can be characterized in the following way. A Banach space $X$ is an Asplund space if and only if each of its separable subspaces has a separable dual (see Phelps (1989)). As an obvious consequence of the Mazur and Asplund results we get

Proposition 3. Let $(X,\|\cdot\|)$ be either a separable Banach space or an Asplund space. Let $C$ be an open convex set in $X$. Then $\mathcal{S}(C)$ is a dense $G_{\delta}$-set in the boundary $\partial C$.

Proof. Let $x_{0} \in C$ and let $f(x)=\inf \left\{t>0:\left(x-x_{0}\right) / t \in C\right\}$ be the Minkowski norm induced by the set $C-x_{0}$. The function $f(x)$ is convex, 
and thus it is Gateaux differentiable on a dense $G_{\delta^{-}}$set $C_{f}$. Since $f(x)$ is positively homogeneous, $\partial C \cap C_{f}$ is a dense $G_{\delta}$-set in $\partial C$.

It is a natural question to which classes of sets Proposition 3 can be extended.

Let $\alpha(\cdot):[0,+\infty) \rightarrow[0,+\infty]$ be a nondecreasing function such that

$$
\lim _{t \downarrow 0} \frac{\alpha(t)}{t}=0 .
$$

Let $f(\cdot)$ be a real-valued continuous function defined on an open convex subset $\Omega \subset X$. We say that $f(\cdot)$ is strongly $\alpha(\cdot)$-paraconvex (Rolewicz (2000)) if for all $x, y \in \Omega$ and $0 \leq t \leq 1$ we have

$$
f(t x+(1-t) y) \leq t f(x)+(1-t) f(y)+\min [t,(1-t)] \alpha(\|x-y\|) .
$$

We say that an open set $C \subset X$ is $\alpha(\cdot)$-intrinsically paraconvex (cf. Ngai-Pénot (2008)) if for all $x, y \in C$,

$$
\operatorname{dist}(t x+(1-t) y, C) \leq t(1-t) \alpha(\|x-y\|) .
$$

Proposition 4. Let $(X,\|\cdot\|)$ be either a separable Banach space or an Asplund space. Let $C$ be an open bounded $\alpha(\cdot)$-intrinsically paraconvex set in $X$. Assume that there is $x_{0} \in C$ such that $C$ is starshaped with respect to $x_{0}$. Then $\mathcal{S}(C)$ is a dense $G_{\delta}$-set in $\partial C$.

Proof. Let $f(x)=\inf \left\{t>0:\left(x-x_{0}\right) / t \in C\right\}$. It is easy to see that there is $c>0$ such that $f(\cdot)$ is strongly $c \alpha(\cdot)$-paraconvex. Thus it is Gateaux differentiable on a dense $G_{\delta}$-set $C_{f}$ (Rolewicz (2002), (2005a), (2006)). Since $f(x)$ is positively homogeneous, $\partial C \cap C_{f}$ is a residual subset of $\partial C$.

Question 5. Is Proposition 4 valid without the assumption that $C$ is starshaped with respect to some point?

By using Proposition 4 we can show this only for locally starshaped intrinsically paraconvex sets.

We say that an open set $C$ is a locally starshaped intrinsically paraconvex set if for any $x_{0} \in \partial C$, there are a neighbourhood $U$ of $x_{0}$ and $\alpha(\cdot)$ satisfying (2) such that $U \cap C$ is an $\alpha$-intrinsically paraconvex set starshaped with respect to some $x_{1} \in U \cap C$.

Proposition 6. Let $(X,\|\cdot\|)$ be either a separable Banach space or an Asplund space. Let $C$ be an open locally starshaped intrinsically paraconvex set in $X$. Then $\mathcal{S}(C)$ is a dense $G_{\delta}$-set in $\partial C$.

Proof. Fix $x_{0} \in \partial C$. By our assumption there are a neighbourhood $U$ of $x_{0}$ and $\alpha(\cdot)$ satisfying $(2)$ such that $U \cap C$ is an intrinsically paraconvex set starshaped with respect to some $x_{1} \in U \cap C$. Thus by Proposition 4,

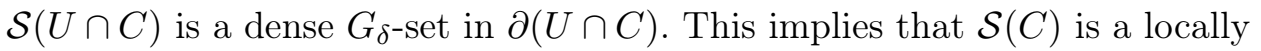


$G_{\delta}$-set in $\partial C$. Therefore by the Michael theorem (Michael (1954)) it is a $G_{\delta}$-set in $\partial C$. Since $x_{0}$ was arbitrary, $\mathcal{S}(C)$ is dense in $\partial C$.

\section{References}

E. Asplund (1966), Farthest points in reflexive locally uniformly rotund Banach spaces, Israel J. Math. 4, 213-216.

E. Asplund (1968), Fréchet differentiability of convex functions, Acta Math. 121, 31-47.

A. Ya. Dubovitskir and A. A. Milyutin (1965), Extremal problems in presence of constraints, Zh. Vychisl. Mat. i Mat. Fiz. 5, 395-453 (in Russian).

S. Mazur (1933), Über konvexe Mengen in linearen normierten Räumen, Studia Math. 4, $70-84$.

E. Michael (1954), Local properties of topological spaces, Duke Math. J. 21, 163-174.

H. V. Ngai and J.-P. Pénot (2008), Paraconvex functions and paraconvex sets, Studia Math. 184, 1-29.

R. R. Phelps (1989), Convex Functions, Monotone Operators and Differentiability, Lecture Notes in Math. 1364, Springer.

S. Rolewicz (2000), On $\alpha(\cdot)$-paraconvex and strongly $\alpha(\cdot)$-paraconvex functions, Control Cybernet. 29, 367-377.

S. Rolewicz (2002), On $\alpha(\cdot)$-monotone multifunctions and differentiability of strongly $\alpha(\cdot)$-paraconvex functions, ibid. 31, 601-619.

S. Rolewicz (2005a), On differentiability of strongly $\alpha(\cdot)$-paraconvex functions in nonseparable Asplund spaces, Studia Math. 167, 235-244.

S. Rolewicz (2005b), Paraconvex analysis, Control Cybernet. 34, 951-965.

S. Rolewicz (2006), An extension of Mazur's theorem on Gateaux differentiability to the class of strongly $\alpha(\cdot)$-paraconvex functions, Studia Math. 172, 243-248.

S. Rolewicz (2007), Paraconvex analysis on $C_{\mathbf{E}}^{1, u}$-manifolds, Optimization 56, 49-60.

Institute of Mathematics

Polish Academy of Sciences

Śniadeckich 8

P.O. Box 21

00-956 Warszawa 10, Poland

E-mail: rolewicz@impan.pl

Received February 9, 2008

Revised version June 19, 2008 